\title{
Prevention of injury by resveratrol in a rat model of adenine-induced chronic kidney disease
}

\author{
Xiaoyan Zhang ${ }^{1}$, Zhenning Yang ${ }^{2}$, Leifang Li $^{1}$, Yanhong Qiao ${ }^{1}$, Haiyan Jiao ${ }^{1}$, \\ Congxiu Miao ${ }^{3 *}$ \\ ${ }^{1}$ Department of Nephrology, Heping Hospital Affiliated to Changzhi Medical College, Changzhi, Shanxi 046000, ${ }^{2}$ Clinical \\ Medicine, Norman Bethune Health Science Center of Jilin University, Changchun, Jinin 130022, ${ }^{3}$ Scientific Research \\ Department, Changzhi Medical College, Changzhi, Shanxi 046000, China
}

*For correspondence: Email: congxiumiao1392@yahoo.com; Tel/Fax: 0086-3553033016

Sent for review: 9 April 2017

Revised accepted: 17 July 2017

\begin{abstract}
Purpose: To investigate the preventive effect of resveratrol against renal pathological changes in a rat model of chronic kidney disease (CKD).

Methods: CKD was induced by daily intragastric administration of adenine $(200 \mathrm{mg} / \mathrm{kg})$ for 1 month. The effect of 10,15 , and $20 \mathrm{mg} / \mathrm{kg}$ doses of resveratrol on the levels of parathyroid hormone, phosphorous, and fibroblast growth factor-23 (FGF-23) in rat urine samples after 2 months of adenine administration were analyzed using an auto-analyzer.

Results: Resveratrol treatment significantly inhibited the adenine-mediated increase in serum parathyroid hormone, phosphorous and FGF-23 levels $(p<0.002)$. In rats treated with 10,15 and 20 $\mathrm{mg} / \mathrm{kg}$ doses of resveratrol after adenine, urine protein/creatinine ratio was reduced to $5,675.6 \pm$ 2453.7, 4, $789.8 \pm 1,534.9$, and 1, $965 \pm 576.8 \mathrm{mg} / \mathrm{g}$, respectively. In the untreated and normal control groups, the respective values were 7, $004 \pm 1,653.3$ and 1, 627.5 $\pm 568.7 \mathrm{mg} / \mathrm{g}$. Treatment with resveratrol after administration of adenine inhibited increases in creatinine, blood urea nitrogen, and uric acid levels in a dose-dependent manner $(p<0.002)$. Resveratrol treatment also inhibited adeninemediated increases in monocytes and inflammatory cells. Furthermore, resveratrol prevented renal tubule swelling and expansion induced by adenine administration.

Conclusion: Resveratrol treatment prevent the renal pathological changes induced by adenine administration in a rat model of CKD by inhibiting FGF-23, parathyroid hormone, and phosphate. Thus, resveratrol may be of therapeutic importance for the treatment of CKD.
\end{abstract}

Keyword: Parathyroid hormone, Phosphate, Creatinine, Monocytes, Chronic kidney disease, Fibroblast growth factor-23

Tropical Journal of Pharmaceutical Research is indexed by Science Citation Index (SciSearch), Scopus, International Pharmaceutical Abstract, Chemical Abstracts, Embase, Index Copernicus, EBSCO, African Index Medicus, JournalSeek, Journal Citation Reports/Science Edition, Directory of Open Access Journals (DOAJ), African Journal Online, Bioline International, Open-J-Gate and Pharmacy Abstracts

\section{INTRODUCTION}

Chronic kidney disease (CKD) is associated with inflammation and hemodynamic changes that result in scarring of the renal tubules and interstitial spaces, and ultimately, the development of end-stage renal disease [1]. CKD is frequently diagnosed and currently represents the leading cause of death among patients with diabetes and hypertension [1].

Studies have shown that a prolonged increase in the serum levels of proteinuria and fibroblast growth factor-23 (FGF-23) is the main cause of end-stage renal disease [2,3]. Production of FGF-23 by bone catalyzes the excretion of inorganic phosphate from the kidneys via the 
urine [4]. FGF-23 also inhibits the generation of vitamin $D$ and maintains the balance between calcium and inorganic phosphate [4]. High levels of inorganic phosphate, parathyroid hormone, and FGF-23 in the serum promote the development of CKD [5]. Another factor that contributes to the development of kidney and heart disease is the presence of high levels of protein in urine [6]. Increased proteinuria interferes with the normal functioning of renal tubules and its suppression may be of therapeutic significance with respect to restoring normal kidney function. Therefore, reducing high levels of proteinuria should be a target for improving renal tubule function, and ultimately, treatment of CKD [6].

Trans-3, 5, 4'-trihydroxystilbene, commonly known as resveratrol, is a polyphenolic compound found in grapes, mulberries, and peanuts. Resveratrol exhibits a wide range of biological activities such as anti-fungal, antiinflammatory, anti-mutagenic, and anti-cancer effects [7]. Resveratrol confers potent antiinflammatory effects by inhibiting cellular inflammatory processes [8], and also inhibits the functions of chondrocytes by targeting the expression of interleukin-1 $\beta$ and inhibiting the generation of reactive oxygen species [9]. Resveratrol treatment also inhibits the production of inducible nitric oxide synthase by human epithelial cells [10]. In addition, it prevents cartilage degradation in animal models of arthritis [11]. This study examined the utility of resveratrol for treating CKD by suppressing the expression of inorganic phosphate, parathyroid hormone, and FGF-23 in a rat model.

\section{METHODS}

\section{Chemicals and reagents}

The adenine and resveratrol used in this study were obtained from Sigma-Aldrich (St. Louis, MO, USA).

\section{Animal study protocols}

Twenty-five male Sprague-Dawley rats, each weighing about $200 \mathrm{~g}$, were obtained from the Experimental Animal Center of Hebei Medical University [animal license: SCXK (Hebei 2015-2034)]. The animal studies were approved by department of nephrology, Heping Hospital affiliated to Changzhi Medical College, Changzhi, Shanxi, China (Approval No: RXT52323). The rats were acclimatized to the laboratory conditions for 1 week before beginning the experiment, and were housed under a 12 $\mathrm{h} / 12 \mathrm{~h}$ light/dark cycle at a constant temperature of $25 \pm 1{ }^{\circ} \mathrm{C}$ and humidity in the range of $50-60$ $\%$. All of the animals had free access to food and water. The experimental protocols used were in accordance with the international suggestions for the Care and Use of Laboratory Animals (NIH publication no. 85-23, revised 1985) [12]. The animals were randomly assigned to five groups of five animals each. The rats in four of the groups (model group and three treatment groups) were administered adenine daily (200 $\mathrm{mg} / \mathrm{kg}$ intragastrically) for 1 month. The three treatment groups were given 10, 15, and 20 $\mathrm{mg} / \mathrm{kg}$ doses of resveratrol daily for 2 months, and the model group received normal saline.

Resveratrol was delivered through the intragastric route, and rats in the normal group received an equal volume of normal saline alone during the treatment period. Completion of treatment was followed by animal sacrifice, with halothane used to extract the kidney and collect the blood samples. At $24 \mathrm{~h}$ before sacrifice, urine was collected from each rat for analysis according to a previously reported protocol [13].

\section{Analysis of kidney damage}

The levels of creatinine and blood urea nitrogen (BUN) in the blood and urine samples were determined using an automatic biochemistry analyzer (Hitachi 7600-020/7170A; Hitachi HighTechnologies Corp., Tokyo, Japan). Briefly, the blood and urine samples collected from the rats were subjected to centrifugation for $15 \mathrm{~min}$ at 1 , $120 \times \mathrm{g}$. The levels of creatinine, BUN, parathyroid hormone, phosphorous, and uric acid in the urine samples, as well as the protein/creatinine ratio, were analyzed using the biochemistry analyzer [13].

\section{Analysis of pathological changes in renal tubules}

The extracted kidneys were washed with phosphate-buffered saline (PBS) and fixed with buffered formalin (10\%), after which they were subjected to paraffin embedding, as previously reported [13]. The paraffin-embedded samples were cut into thin $(2 \mu \mathrm{m})$ sections and boiled in xylene solution. The sections were stained with hematoxylin and eosin to analyze changes in renal tubule morphology under a magnification of $400 x$. Three regions of each section were examined randomly.

\section{Analysis of serum fibroblast growth factor-23}

The levels of FGF-23 in rat serum samples were analyzed using an FGF-23 enzyme-linked immunosorbent assay (ELISA) kit (Immutopics, 
San Clemente, CA, USA) according to the manufacturer's protocol. Briefly, the serum samples were subjected to centrifugation for 10 min at $1,500 \mathrm{rpm}$ and subsequently stored at -20 ${ }^{\circ} \mathrm{C}$ for ELISA analysis. The serum samples were subjected to culture for $24 \mathrm{~h}$ after being added to 96-well microplates pre-coated with mouse monoclonal FGF-23 antibodies. After culture, the plates were washed and treated with horseradish peroxidase-conjugated polyclonal FGF-23 secondary antibodies. Then the plates were washed again and treated with $\mathrm{H}_{2} \mathrm{O}_{2}$ and tetramethylbenzidine followed by measurement of absorbance at $450 \mathrm{~nm}$.

\section{Statistical analysis}

The data are presented as means \pm SD for each rat group. Analysis of the statistical differences between both groups of rats was done using unpaired Student's $t$-tests. Differences among all groups were analyzed by one-way analysis of variance with Bonferroni correction applied. All of the data were analyzed using SPSS software (ver. 12.0; SPSS, Inc., Chicago, IL, USA). $P<$ 0.05 was taken as statistically significant.

\section{RESULTS}

Effects of resveratrol treatment on adeninemediated upregulation of FGF23, inorganic phosphate, and parathyroid hormone in rat serum

Adenine administration markedly increased the levels of FGF-23, inorganic phosphate, and parathyroid hormone in rat serum compared to those of the control group $(p<0.005)$. However, treatment with resveratrol after adenine administration significantly reduced the increases in serum FGF-23, inorganic phosphate, and parathyroid hormone relative to the model control group (Figure 1). The serum levels of FGF-23 and parathyroid hormone were very similar between the normal and resveratrol-treated (20 $\mathrm{mg} / \mathrm{kg}$ ) rats.

\section{Effects of resveratrol treatment on adenine- mediated increase in urine protein/creatinine ratio}

In rats administered adenine, the urine protein/creatinine ratio was markedly increased compared to that of the normal group. Treatment with resveratrol after adenine significantly attenuated this increase in the protein/creatinine ratio $(p<0.001)$. In the rats treated with 10,15 , and $20 \mathrm{mg} / \mathrm{kg}$ doses of resveratrol after adenine administration, the urine protein/creatinine ratio was reduced significantly, to $5,675.6 \pm 2$, 453.7, $4,789.8 \pm 1,534.9$ and $1,965 \pm 576.8 \mathrm{mg} / \mathrm{g}$, respectively, compared to values in the model control group (7, $004 \pm 1,653.3)$. The urine protein/creatinine ratio of the normal control group was 1, $627.5 \pm 568.7 \mathrm{mg} / \mathrm{g}$ (Figure 2). The increase in uric acid level caused by adenine administration was also significantly reduced by treatment with resveratrol in all three dose groups $(p<0.002)$.

Resveratrol treatment lowered the adeninemediated increase in serum creatinine and BUN levels

In rats administered adenine, the serum levels of creatinine and BUN were markedly increased compared to those of the normal group ( $p<$ 0.002). Treatment with resveratrol after administration of adenine attenuated the increases in creatinine and BUN levels in a dosedependent manner (Figure 3).

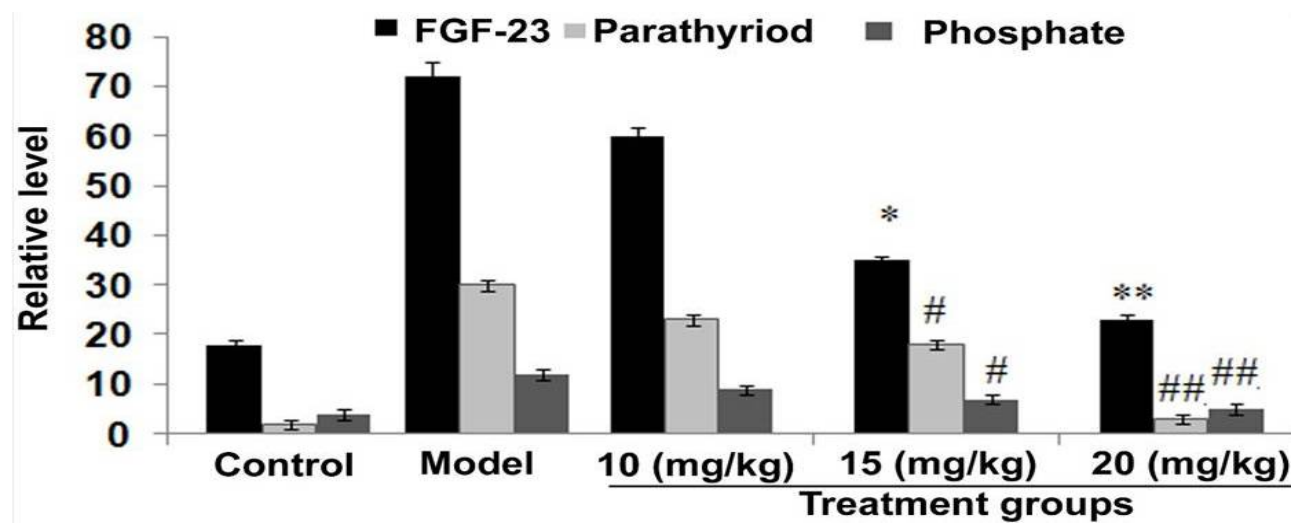

Figure 1: Effects of resveratrol on adenine-induced increases in fibroblast growth factor-23 (FGF-23), inorganic phosphate, and parathyroid hormone levels. Rats in the treatment groups were given 10, 15 , and $20 \mathrm{mg} / \mathrm{kg}$ doses of resveratrol after adenine administration, whereas the normal rats only received saline. The control group was given normal saline after adenine administration; ${ }^{*} p<0.05,{ }^{* *} p$ $<0.01, \# p<0.05$, and $\# \# p<0.01$ compared to the model control group 

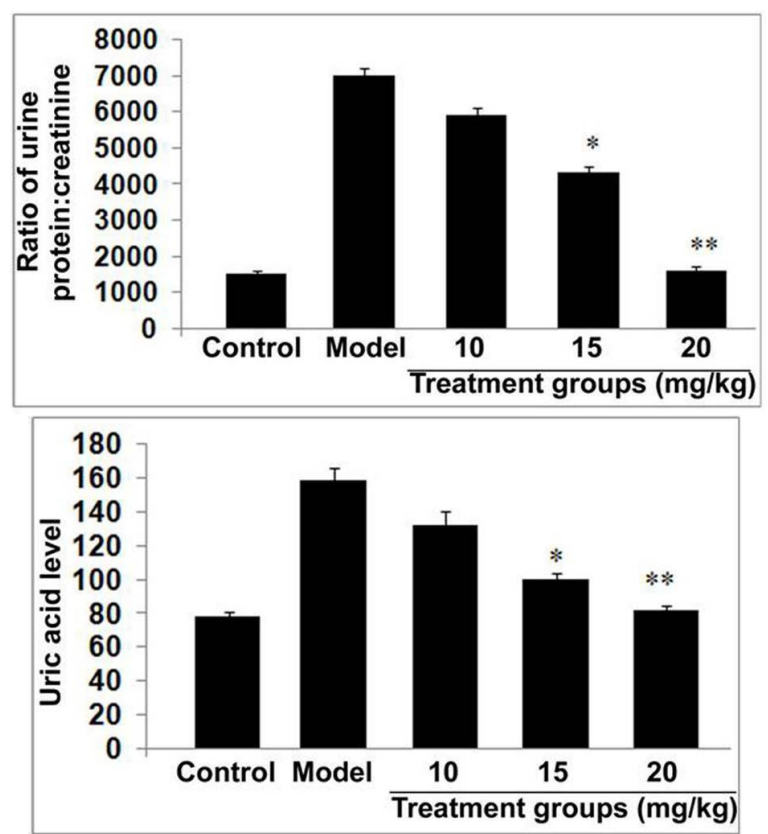

Figure 2: Effects of resveratrol treatment on the adenine-mediated increase in the urine protein/creatinine ratio. Rats administered adenine were treated with 10,15 , and $20 \mathrm{mg} / \mathrm{kg}$ doses of resveratrol or normal saline (control). The urine of the rats was collected over a $24 \mathrm{~h}$ period to determine the levels of protein and creatinine; ${ }^{*} p<0.05$ and ${ }^{* *} p<0.01$ compared to the untreated control group

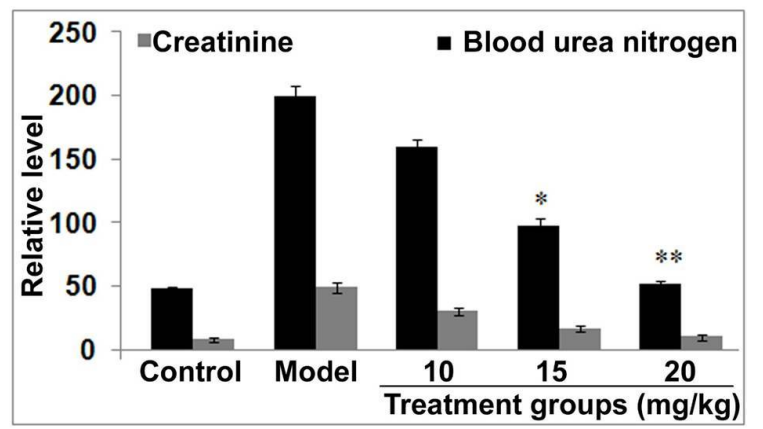

Figure 3: Effects of resveratrol treatment on levels of creatinine and blood urea nitrogen (BUN). Rats administered adenine were treated with 10,15 , and $20 \mathrm{mg} / \mathrm{kg}$ doses of resveratrol or normal saline (control). The serum levels of creatinine $(\mu \mathrm{mol} / \mathrm{L})$ and $\mathrm{BUN}(\mathrm{mmol} / \mathrm{L})$ were analyzed; ${ }^{*} p<0.05,{ }^{* *} p<0.01$, \#p $<0.05$ and $\# \# p<0.01$ compared to the untreated control group

Resveratrol treatment prevents adenineinduced pathological changes in rat kidney

Administration of adenine caused changes in the renal tubules in the kidneys of the rats. Examination of the renal tubules of rats administered adenine revealed marked swelling and expansion $(p<0.005)$. The tubules in the adenine groups had higher levels of dihydroxyadenine than normal control rats, and the renal tissues also had marked increases in monocytes and inflammatory cells $(p<0.002$; Figure 4). Resveratrol treatment inhibited the adenine-mediated increase in monocytes and inflammatory cells and prevented renal tubule swelling and expansion (Figure 4).

\section{DISCUSSION}

Acute kidney injury is encountered frequently during clinical practice and affects the survival of patients. Injury to the renal tubules leads to acute renal failure in more than $80 \%$ of patients $[14,15]$. This study demonstrated curative effects of resveratrol on the renal pathological changes occurring in a rat model of CKD, in which the animals were administered adenine intragastrically.

Levels of phosphorus and calcium in the kidneys are regulated and maintained by various hormones such as parathyroid hormone, calcitriol, and FGF-23 [16]. Upregulation of phosphorus, FGF-23, and parathyroid hormone is associated with worsening kidney disease and a higher likelihood of death $[17,18]$. This study demonstrated a marked decrease in adenineinduced increases in serum phosphorus, FGF23, and parathyroid hormone levels in rats, suggesting the preventative effects of resveratrol against adenine-mediated kidney disorders. The increased urine protein/creatinine ratio caused by adenine administration was also attenuated by treatment with resveratrol. Furthermore, our study demonstrated that rats administered adenine had higher serum levels of creatinine, BUN, and uric acid. Treatment of rats with resveratrol after administration of adenine inhibited the increases in creatinine, BUN, and uric acid levels in a dose-dependent manner.

The results of this study showed that adenine administration caused changes in the renal tubules in the kidneys of rats; there was considerable swelling and expansion of the renal tubules. The level of dihydroxyadenine in the renal tubules of rats administered adenine was markedly higher than that of the normal control rats, and the renal tissues also had markedly increased levels of monocytes and inflammatory cells. However, resveratrol treatment inhibited the adenine-mediated increase in monocytes and inflammatory cells and prevented renal tubule swelling and expansion. 
Control

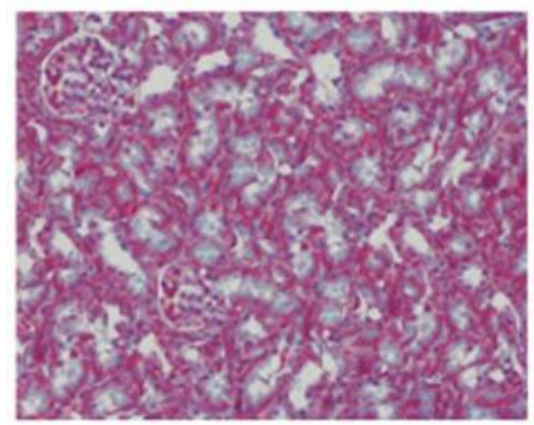

Adenine

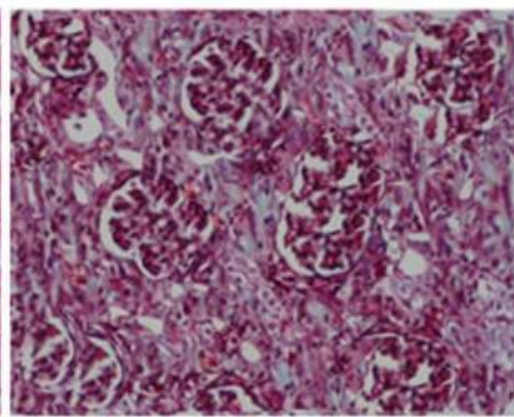

Adenine+Resv. $(10 \mathrm{mg} / \mathrm{kg})$

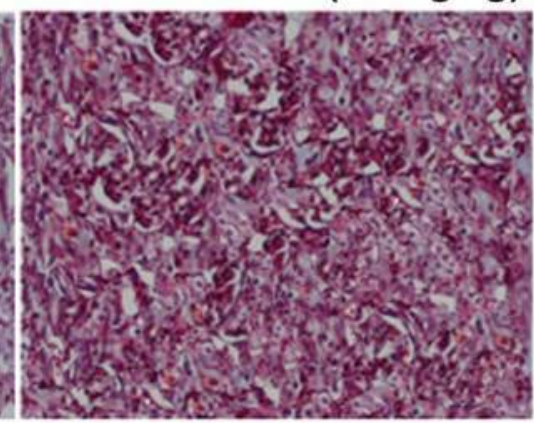

Adenine+Resv. $(15 \mathrm{mg} / \mathrm{kg})$ Adenine+Resv. $(20 \mathrm{mg} / \mathrm{kg})$
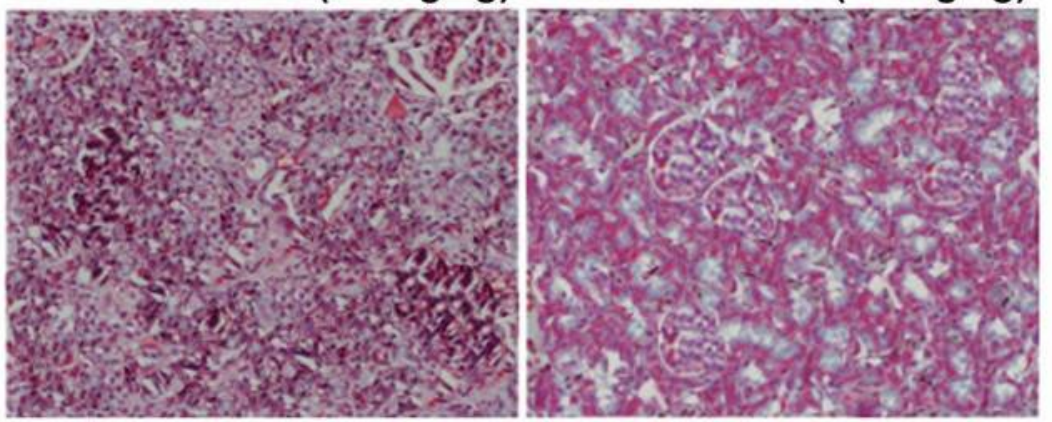

Figure 4: Resveratrol treatment prevented adenine-induced pathological changes in the rat kidney. Rats administered adenine were treated with resveratrol at 10, 15, and $20 \mathrm{mg} / \mathrm{kg}$ doses or normal saline (control). Renal tissues were examined for pathological changes after staining with hematoxylin and resveratrol.

\section{CONCLUSION}

Resveratrol treatment prevents renal pathological changes induced by adenine administration in a CKD rat model by inhibiting FGF-23, parathyroid hormone, and phosphate. Thus, resveratrol may have therapeutic importance for the treatment of CKD.

\section{DECLARATIONS}

\section{Acknowledgement}

The authors are thankful to the Department of Nephrology, Affiliated Heping Hospital of Changzhi Medical College, Shanxi, China, for financial support.

\section{Conflict of Interest}

No conflict of interest associated with this work.

\section{Contribution of Authors}

The authors declare that this work was done by the authors named in this article and all liabilities pertaining to claims relating to the content of this article will be borne by them.

\section{Open Access}

This is an Open Access article that uses a funding model which does not charge readers or their institutions for access and distributed under the terms of the Creative Commons Attribution License (http://creativecommons.org/ licenses/by/ 4.0) and the Budapest Open Access Initiative (http://www.budapestopena ccessinitiative.org/read), which permit unrestricted use, distribution, and reproduction in any medium, provided the original work is properly credited.

\section{REFERENCES}

1. Noronha IL, Fujihara CK, Zatz R. The inflammatory component in progressive renal disease $\square$ are interventions possible? Nephrol Dial Transplant 2002;

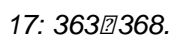

2. Sarnak MJ, Astor BC. Implications of proteinuria: $C K D$ progression and cardiovascular outcomes. Adv Chronic Kidney Dis 2011; 18: 258-266.

3. Faul C. Fibroblast growth factor 23 and the heart. Curr Opin Nephrol Hypertens 2012; 21: 369@375.

4. Razzaque MS, Lanske $B$. The emerging role of the fibroblast growth factor-23-klotho axis in renal regulation 
of phosphate homeostasis. J Endocrinol 2007; 194: 1 1010.

5. Seiler S, Heine GH, Fliser D. Clinical relevance of FGF23 in chronic kidney disease. Kidney Int Suppl 2009; 76. S34⿴囗口 42 .

6. Sharma SK, Zou $H$, Togtokh A, Ene-lordache $B$, Carminati S, Remuzzi A, Wiebe N, Ayyalasomayajula B, Perico N. Burden of CKD, proteinuria, and cardiovascular risk among Chinese, Mongolian, and Nepalese participants in the International Society of Nephrology screening presveratrolrams. Am J Kidney Dis 2010; 56: 915回927.

7. Jang M, Cai L, Udeani GO, Slowing KV, Thomas CF, Beecher CWW, Fong HHS, Farnsworth NR, Kinghorn $A D$, Mehta RG, Moon RC, Pezzuto JM. Cancer chemopreventive activity of resveratrol, a natural product derived from grapes. Science 1997; 275: 218220.

8. Bishayee A, Waghray A, Barnes KF, Mbimba T, Bhatia D, Chatterjee M, Darvesh AS. Suppression of the inflammatory cascade is implicated in resveratrol chemoprevention of experimental hepatocarcinogenesis. Pharm Res 2010; 27: 10801091.

9. Knobloch $J$, Sibbing $B$, Jungck $D$, Lin $Y$, Urban $K$, Stoelben E, Strauch J, Koch A. Resveratrol impairs the release of steroid]resistant inflammatory cytokines from human airway smooth muscle cells in chronic obstructive pulmonary disease. J Pharmacol Exp Ther 2010; 335: 788-798.

10. Chung EY, Kim BH, Hong JT, Lee CK, Ahn B, Nam SY, Han SB, Kim Y. Resveratrol downregulates interferongamma-inducible inflammatory genes in macrophages: molecular mechanism via decreased STAT-1 activation. J Nutr Biochem 2011; 22: 902-909.

11. Csaki C, Keshishzadeh N, Fischer K, Shakibaei M. Regulation of inflammation signalling by resveratrol in human chondrocytes in vitro. Biochem Pharmacol 2008; 75: 677-687.

12. "Principles of Laboratory Animal Care" (NIH publication no. 85-23, revised 1985). Available from: http://grants 1.nih.gov/grants/olaw/references/phspol.htm

13. H, Yan L, Zhihua Z, Xiaohua W, Mingliang H, Rongjun $L$, Xueqing $Y$. Rosiglitazone alleviates injury in rats with adenine?induced chronic kidney disease. Mol Med Rep 2013; 8: 1831-1835.

14. Donnelly LE, Newton R, Kennedy GE, Fenwick PS, Leung $R H$, Ito $K$, Russell RE, Barnes PJ. Anti]inflammatory effects of resveratrol in lung epithelial cells: molecular mechanisms. Am J Physiol Lung Cell Mol Physiol 2004; 287: L774-L783.

15. Singbartl K, Kellum JA. AKI in the ICU: Definition, epidemiology, risk stratification, and outcomes. Kidney Int 2012; 81: 819-825.

16. Bergwitz $C$, Jüppner $H$. Regulation of phosphate homeostasis by PTH, vitamin D, and FGF23. Annu Rev Med 2010; 61: 910104.

17. Pontoriero G, Cozzolino M, Locatelli F, Brancaccio $D$. CKD patients: the dilemma of serum PTH levels. Nephron Clin Pract 2010; 116: c263-c268.

18. Levin A, Djurdjev O, Beaulieu M, Er L. Variability and risk factors for kidney disease progression and death following attainment of stage 4 CKD in a referred cohort. Am J Kidney Dis 2008; 52: 661⿴671. 